\title{
The emerging role of PTBP1 in human cancer: novel prognostic factor in non-muscle invasive bladder cancer
}

\author{
Pamela Bielli ${ }^{1,2}$, Savino M. Di Stasi ${ }^{3}$, Claudio Sette ${ }^{2,4}$ \\ ${ }^{1}$ Department of Biomedicine and Prevention, University of Rome Tor Vergata, Rome, Italy; ${ }^{2}$ Fondazione Santa Lucia IRCCS, Rome, Italy; \\ ${ }^{3}$ Department of Experimental Medicine and Surgery, University of Rome Tor Vergata, Rome, Italy; Institute of Human Anatomy and Cell Biology, \\ Università Cattolica del Sacro Cuore, Rome, Italy \\ Correspondence to: Claudio Sette. Institute of Human Anatomy and Cell Biology, Catholic University of the Sacred Hearth, Mail, Italy. \\ Email: claudio.sette@unicatt.it. \\ Provenance: This is an invited article commissioned by Section Editor Xiao Li (Department of Urology, Jiangsu Cancer Hospital \& Jiangsu Institute \\ of Cancer Research \& Nanjing Medical University Affiliated Cancer Hospital, Nanjing, China). \\ Response to: Lee KH, Ku JH. Targeting splicing factors as molecular non-muscle invasive bladder cancer predictors. Transl Androl Urol 2018. doi: \\ $10.21037 /$ tau. 2018.10 .09
}

Submitted Nov 21, 2018. Accepted for publication Dec 01, 2018.

doi: $10.21037 /$ tau.2018.12.01

View this article at: http://dx.doi.org/10.21037/tau.2018.12.01

Alternative splicing is a sophisticated RNA processing mechanism that expands the coding potential of the genome and the proteomic complexity in higher eukaryotes. Splicing requires the recognition of the intron/exon boundaries by a large ribonucleoprotein complex, named the spliceosome, followed by intron excision and ligation of exons to yield the messenger RNA (mRNA) (1). Since splice sites sequences are short and degenerate, the precise recognition of the exon-intron boundary requires binding of additional trans-acting splicing factors to intronic and exonic sequence-specific motifs, which cooperate with the spliceosome in determining the final splicing outcome $(1,2)$. Indeed, competition between antagonistic splicing factors for sequences in the nascent pre-mRNA influences splicing of a large number of exons, allowing most human genes to yield multiple splice variants in a context-specific fashion (3). Nevertheless, such flexible process is prone to errors and dysregulation of alternative splicing underlies many human diseases, including cancer (2). Of note, recent high-throughput transcriptome analyses have demonstrated that many types of human tumors, including bladder cancer, display a specific "splicing signature", which often exhibit prognostic value and may represent a cancer-specific therapeutic target in patients (4).

Dysregulation of splicing patterns in cancer may result as a consequence of somatic mutations in regulatory sequences or from aberrant expression/activity of specific splicing factors $(2,5)$. In this regard, several of these RNA-binding proteins have been shown to impact on tumorigenesis by directly affecting specific gene expression programs. Among them, PTBP1 is emerging as a powerful prooncogenic factor in multiple human cancer types. PTBP1 is an essential protein and knockout of the gene encoding this protein in the mouse leads to embryonic lethality (6), indicating crucial and non-redundant functions played by PTBP1 in the cell. On the other hand, its up-regulation confers increased malignancy in cancer. The pro-oncogenic function of PTBP1 was first reported in gliomas (7), where it contributes to tumor progression by modulating alternative splicing (8). Following these initial reports, high levels of PTBP1 were confirmed to associate with aggressive behavior of other types of cancer. In many cases, PTBP1 was reported to exert pro-tumorigenic effects on proliferation, survival, senescence and resistance to chemotherapy by modulating the splicing of cancer-relevant genes, such as fibroblast growth factor receptor-1 and 2, USP5, alpha-actinin and PKM. Furthermore, PTBP1 plays multiple roles in post-transcriptional regulation of gene expression, including regulation of microRNA biogenesis, mRNA translation and stability. These observations suggest that even a slight alteration of its expression levels may profoundly impact on the regulation of a wide gene expression program, with likely consequences on multiple biological processes. However, since most, if not all, the 
functions of PTBP1 appear to be dependent on its ability to specifically bind RNA, targeting its RNA-binding activity may represent a suitable therapeutic approach for human cancers in which this splicing factor contributes to tumorigenesis.

Non-muscle invasive bladder cancer (NMIBC) represents the most common and heterogeneous malignancy of the urinary tract. Due to its high recurrence probability and progression rate, the mortality rate and the management costs of NMIBC patients remain high. Despite strong efforts in the last decades, the progress in the management of newly diagnosed NMIBC remains modest (9). Thus, identification of robust prognostic markers for predicting the clinical outcome and improving therapeutic approaches stands as a major concern for clinicians. In this scenario, expression of pro-oncogenic splice variants of several genes was previously reported also in bladder cancer and it was shown to correlate with disease progression. However, whether splicing dysregulation was caused by specific splicing factors and whether their expression displayed prognostic value remained unclear. To directly address this issue, we performed an unbiased bioinformatics analysis of public databases containing clinical and molecular datasets of patients diagnosed with bladder cancer. In particular, we queried whether the expression of selected splicing factors with known oncogenic relevance was associated with relapse-free survival (10). Among the 17 splicing factors tested, we identified PTBP1 as a protein possibly linked to NMIBC prognosis and disease progression. The insilico results were then confirmed by immunohistochemical analysis of specimens from a large cohort of NMIBC patients for whom we collected detailed history and followup data. Next, since expression of pro-oncogenic splice variants modulated by PTBP1 in other cellular contexts has been associated also with bladder cancer progression, we investigated the function of PTBP1 in the regulation of these cancer-relevant genes (10). By using three different bladder cancer cell lines, we showed that PTBP1 directly promoted the oncogenic splice variants of these genes by binding to their pre-mRNA in proximity of the regulated exons. Moreover, for at least one of these genes (i.e., CD44), we observed a positive correlation between PTBP1 expression and inclusion of variable exons also in specimens obtained from NMIBC patients, suggesting that dysregulation of CD44 splicing in bladder cancer patients may be directly caused by altered expression of PTBP1. Collectively, our work highlights a role of PTBP1 in NMIBC progression and suggests that its function is mediated by modulation of alternative splicing of bladder cancer-relevant genes (10).

Our experimental plan did not allow us to test the prognostic value of PTBP1 expression also in longitudinal studies. Nevertheless, although further research is required to completely identify the mechanisms underlying splicing dysregulation in bladder cancer, our work suggests that PTBP1 and its splicing signature are involved in disease progression and could represent novel predictor markers for NMIBC. Since depletion of PTBP1 strongly limited bladder cancer cell malignancy, it is possible that targeting PTBP1, or its splicing program, will represent the basis for the development of novel therapeutics strategies to treat NMIBC.

\section{Acknowledgements}

Funding: The research was supported by the Associazione Italiana Ricerca sul Cancro (AIRC; IG18790) and by Italian Ministry of Health "Ricerca Finalizzata 2011" (GR-201102348423).

\section{Footnote}

Conflicts of Interest: The authors have no conflicts of interest to declare.

\section{References}

1. Matera AG, Wang Z. A day in the life of the spliceosome. Nat Rev Mol Cell Biol 2014;15:108-21.

2. Paronetto MP, Passacantilli I, Sette C. Alternative splicing and cell survival: from tissue homeostasis to disease. Cell Death Differ 2016;23:1919-29.

3. Fu XD, Ares M Jr. Context-dependent control of alternative splicing by RNA-binding proteins. Nat Rev Genet 2014;15:689-701.

4. Lee SC, Abdel-Wahab O. Therapeutic targeting of splicing in cancer. Nat Med 2016;22:976-86.

5. Dvinge H, Kim E, Abdel-Wahab O, et al. RNA splicing factors as oncoproteins and tumour suppressors. Nat Rev Cancer 2016;16:413-30.

6. Shibayama M, Ohno S, Osaka T, et al. Polypyrimidine tract-binding protein is essential for early mouse development and embryonic stem cell proliferation. FEBS J 2009;276:6658-68.

7. Cheung HC, Hai T, Zhu W, et al. Splicing factors PTBP1 and PTBP2 promote proliferation and migration of glioma cell lines. Brain 2009;132:2277-88. 
8. Ferrarese R, Harsh GR 4th, Yadav AK, et al. Lineagespecific splicing of a brain-enriched alternative exon promotes glioblastoma progression. J Clin Invest 2014;124:2861-76.

9. Kamat AM, Hegarty PK, Gee JR, et al. International Consultation on Urologic Disease-European Association of Urology Consultation on Bladder Cancer 2012. ICUDEAU International Consultation on Bladder Cancer 2012:
Screening, diagnosis, and molecular markers. Eur Urol 2013;63:4-15.

10. Bielli P, Panzeri V, Lattanzio R, et al. The Splicing Factor PTBP1 Promotes Expression of Oncogenic Splice Variants and Predicts Poor Prognosis in Patients with Non-muscle-Invasive Bladder Cancer. Clin Cancer Res 2018;24:5422-32.
Cite this article as: Bielli P, Di Stasi SM, Sette C. The emerging role of PTBP1 in human cancer: novel prognostic factor in non-muscle invasive bladder cancer. Transl Androl Urol 2018;7(Suppl 6):S765-S767. doi: 10.21037/tau.2018.12.01 